\title{
Using Micro-Computed Tomography to Investigate Powder Distribution Trends Within 3D Binder-Jet Printed SS316 Parts
}

\author{
Erica Stevens $^{1}$, John Barnes ${ }^{2}$ and Markus Chmielus ${ }^{1}$ \\ 1. Department of Mechanical Engineering and Materials Science, University of Pittsburgh, Pittsburgh, \\ PA 15260 \\ 2. The Barnes Group Advisors, Pittsburgh, PA 15143
}

Binder-jet 3D printing builds parts by spreading successive layers of powder and selectively depositing binder to bind particles in the desired arrangement for each layer. Post-processing for binder-jet printed parts typically includes a curing step at lower temperature and a sintering step at higher temperature. The curing step causes the parts to be stable enough to handle gently.

Though the technology has progressed significantly since its inception, the details of powder particle distribution and spreading are complicated and not completely understood. However, distribution and spreading affects the powder packing and therefore sintering behavior [1]. There have been efforts to model the physics of powder spreading, mostly for the purposes of laser melting techniques, but every mathematical model necessarily makes some idealistic assumptions [2]. In practice, the distribution of powder particle sizes in an actual binder-jet printed part have not been directly examined.

This experiment uses micro-computed tomography to directly view and analyze the powder particles in a cured binder-jet printed SS316 fabricated by the ExOne® Corporation on an M-Flex and made in different sections of the build box. Powder supplied by Hoganas was gas-atomized with a size range of $20-53 \mu \mathrm{m}$. Samples had a diameter of $2 \mathrm{~mm}$ and height $10 \mathrm{~mm}$ and were printed with the circular crosssection in the horizontal plane (plane of movement for the print head). For analysis, samples were scanned with a Bruker Skyscan 1272 from Microphotonics. Reconstruction, analysis, and visualization was performed in Microphotonics software: NRecon, Dataviewer, CTAn, and CTVox. The goals of analysis were: (1) determine whether there was a different in particle size distribution based on sample location in the build box, (2) examine the particle size distribution within a single sample for abnormalities.

Scanning was conducted at $100 \mu \mathrm{A}, 100 \mathrm{kV}$ with a $0.11 \mathrm{~mm} \mathrm{Cu}$ filter and a $1 \mu \mathrm{m}$ square pixel size. After reconstruction and thresholding, it was clear that most particles were touching others, making analysis of individual particles impossible without further processing. Since particles were spherical, it was possible to use the watershed function in CTAn to separate particles. Ideally, the separation would occur in 3D to account for the entire sphere as a whole. However, computing power limited the separation to a $2 \mathrm{D}$ operation, which made for less smooth edges to particles.

Following watershed separation, an individual particle analysis was executed. Due to some improper watershed results, not all particles were valid. Using the knowledge that all particles were expected to be spherical and the results of particle size distribution obtained from laser measurements, individual measurements were filtered to include only particles that had a sphere-equivalent diameter (ESDv) greater than or equal to $12 \mu \mathrm{m}$ and a sphericity of at least 0.7 . This provided approximately 100 valid particles for each sample. There was found to be a bias for the smaller-size particles compared to laser- 
measured results. This is likely due to the watershed operation cutting into larger particles with satellites, which are then eliminated by the sphericity filter.

Individual sample particle distribution was analysed by using a cross-sectional view through height and the watershed particles were colorized according to their size. Understanding that not all particles were bisected, this still would show any significant difference in particle size throughout the height. Though this was done in several locations throughout each sample, no anomalous variations were observed.

\section{References:}

[1] Y Bai, G Wagner, C Williams, J. Manuf. Sci. Eng. 139 (2017), p. 1-6.

[2] M Markl, C Korner, Annu. Rev. Mater. Res. 46 (2016), p. 1-34.

[3] E. Stevens was partially supported by the Department of Defense through the National Defense Science \& Engineering Graduate Fellowship (NDSEG).
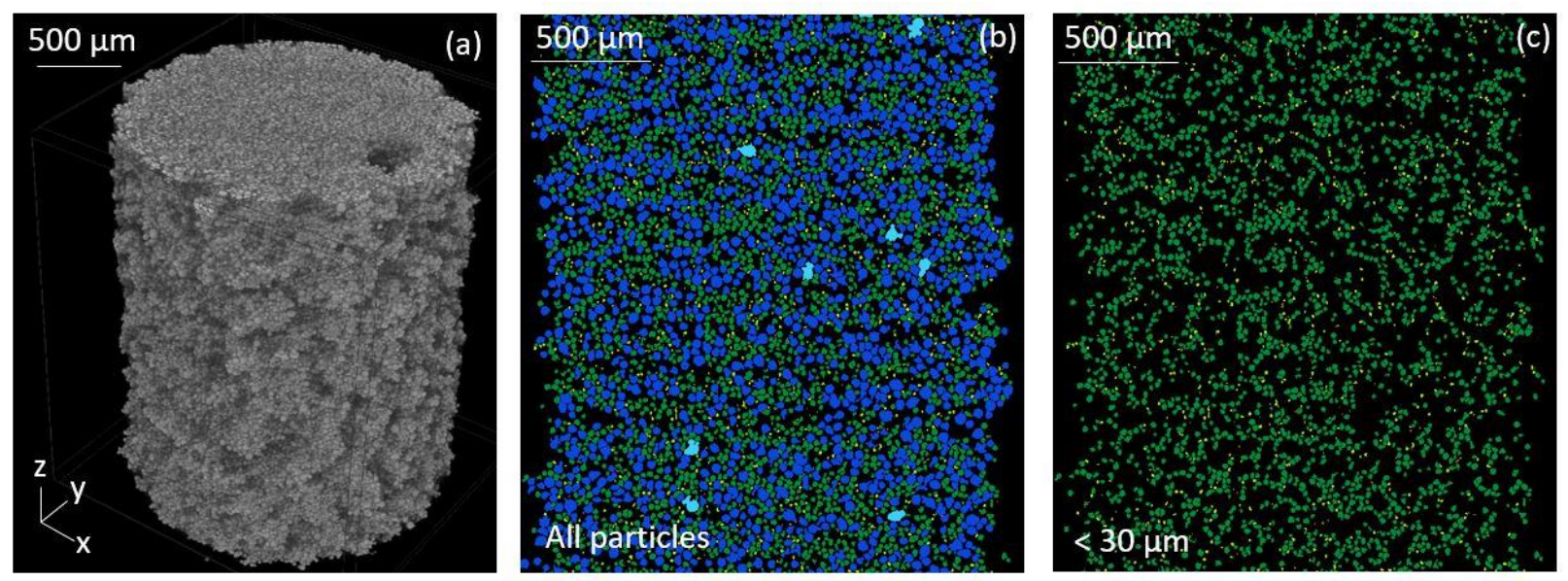

Figure 1. (a) 3D representation of the scanned piece. (b) Cross-section with $\mathrm{z}$ vertical, colorized with ESDv $>0.3 \mu \mathrm{m}$ blue and ESDv $\leq 0.3 \mu \mathrm{m}$ green. (c) Same as (b) but with blue particles removed.

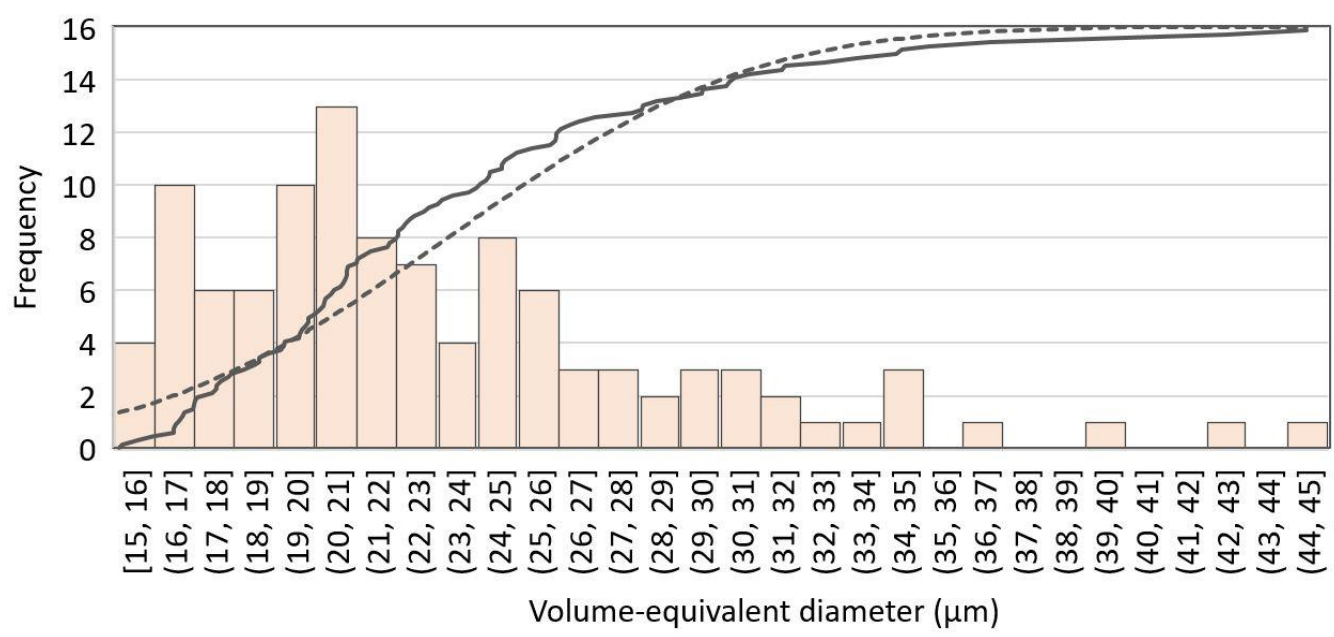

100

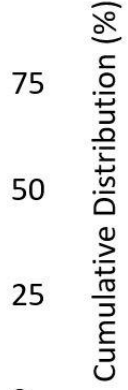

0 